\title{
Sudden Cardiac Death After ECT
}

\author{
Ole J. Thienhaus* \\ Department of Psychiatry, University of Arizona College of Medicine, Tucson, Arizona, USA
}

"Corresponding Author: Ole J. Thienhaus, Department of Psychiatry, University of Arizona College of Medicine, Tucson, Arizona, USA, Tel: +520 626 3261; Email: ojt@ psychiatry.arizona.edu

Received: 07 September 2017; Accepted: 26 September 2017; Published: 30 September 2017

\begin{abstract}
Electroconvulsive therapy is known to be one of the safest methods of treating major depressive episodes. One of the rare complications attributable directly to ECT, rather than general anesthesia, concerns cardiac death in vulnerable patients. The case presented here raises the issue that routine work-up can miss warning signs of cardiac pathology in high risk patients. A history of cocaine use disorder suggests echocardiography to rule out cardiomegaly.
\end{abstract}

Keywords: Electroconvulsive therapy; Echocardiography; Psychopharmacotherapies; Psychiatric; Neurologic

\section{Introduction}

Electroconvulsive therapy (ECT) has established itself as a safe and effective treatment option after its use declined temporarily with the introduction of various psychopharmacotherapies for mood disorders [1]. Overall mortality of patients who receive ECT has been reported to be 0.03 percent [2].

Appropriate psychiatric and neurologic indications have been identified [3]. Techniques have been standardized and there is a fair amount of evidence for its effectiveness in inducing remission of major mood disorders. Concerns about adverse effects have mostly focused on the possibility of cognitive deterioration [4]. The actual safety of the procedure has always been seen as a tremendous asset, making ECT an excellent choice in certain higher risk patients such as patients with hydrocephalus or pregnant women who may not be good candidates for antidepressant or mood-stabilizing pharmacotherapy. Much of the low mortality associated with ECT is due to complications related to the anesthesia rather than to the procedure itself. Careful attention to preexisting cardiac conditions, electrolyte abnormalities and intracranial pathology has been crucial in making ECT so safe. 


\section{Case Report}

In this case report, we want to present a case of a 35 year old man who died of asystole following a maintenance ECT procedure.

The patient had a well-established diagnosis of bipolar disorder, the most recent episode having been depressed. He came from a broken home, essentially being on his own as of age six, getting involved in substance use as a preteen. He used alcohol, opiates and stimulants extensively until about two years prior to his course of ECT. He continued using marijuana regularly. He was proud of his physical stamina and reported that he regularly cut down trees and chopped wood.

He had been in outpatient treatment for his bipolar disorder and tried on a variety of medications including aripiprazol, quetiapine, olanzapine, risperidone, divalproex, carbamazepine, lithium and lamotrigine. None of these medications induced sustained mood stabilization. The patient had a number of brief inpatient stays, the most recent one about a year prior to his ECT. The exact number of hospitalizations is unclear. He had had a record of shortlived employment and was eventually placed on supplemental security income.

The patient was referred for ECT by his primary psychiatrist because his mood disorder had proved refractory to other interventions. He was evaluated and found to meet diagnostic criteria for a major depressive episode as part of a bipolar disorder. At the time of ECT, the patient was maintained on olanzapine, $20 \mathrm{mg}$ per day for mood stabilization, and trazodone $100 \mathrm{mg}$ for sleep induction.

With a body mass index of $36.1 \mathrm{~kg} / \mathrm{m}^{2}$, he was obese and he had a history of hypertension. Otherwise, his physical examination was unremarkable. His pre-ECT work-up included all recommended studies i.e. chest film, EKG, urinalysis, complete blood count, blood glucose and urea nitrogen and serum electrolytes. All results were within normal limits. The patient was classified as ASA II because of his smoking ( 2 packs per day) and his obesity, but he was seen as a safe candidate for general anesthesia and ECT.

ECTs were initiated on an outpatient basis at a frequency of two procedures per week. For each treatment, the patient was prepared by administering $250 \mathrm{mg}$ of caffeine to lower the seizure threshold, followed by $20 \mathrm{mg}$ of etomidate and $120 \mathrm{mg}$ of succinylcholine. The equipment used was a Thymatron machine. In line with the manufacturer's recommendation, the power output was set at $20 \%$ of maximum. The patient generally had well defined, generalized attenuated tonic-clonic muscle phenomena indicating seizure lasting for 15 to 50 seconds. In the course of 13 biweekly ECTs, his postictal recovery had been uneventful each time, and the patient had reported improvement in his depressed mood. The plan was, after the 14th treatment, to reduce frequency to a twice a month schedule.

On the day of the 14th treatment, the patient was prepared the same way as on all previous occasions. His EKG, vital signs and serum electrolytes were all within normal limits. After administration of the bitemporal stimulus, the patient had a 15 second clinically visible response. After the treatment, he appeared to stabilize but then developed 
progressive bradycardia and, despite repeated doses of atropine and epinephrine the bradycardia ended up in asystole. External resuscitation was attempted, but after 45 minutes the patient was pronounced dead.

The patient came to autopsy. His toxicology revealed ephedrine $(1,100 \mathrm{ng} / \mathrm{ml})$ and, unsurprisingly, cannabis metabolites ( $\delta$-9-tetrahydrocannabinol and $\delta$-9-carboxy tetrahydrocannabinol at concentrations of 1.7 and $28.0 \mathrm{ng} / \mathrm{ml}$ respectively), but was otherwise negative. The cannabis metabolites were consistent with the known use of marijuana. The ephedrine concentration far exceeded that seen after therapeutic use of standard ephedrinecontaining decongestants (up to $100 \mathrm{ng} / \mathrm{ml}$ ) and suggests excessive use of such compounds as a substitute of his abandoned methamphetamine and cocaine abuse.

He was found to have cardiomegaly with his heart weighing 570 grams (average weight of a male heart is 300 grams), and hypertrophy of his left ventricle.

The heart is definitely coming under stress while ECT is administered. Cardiac workload initially increases with the onset of the seizure due to sympathetic stimulation originating in the diencephalon [5]. As the seizure progresses, circulating catecholamine levels rise [6]. After the seizure terminates, parasympathetic rebound commences and results in the commonly observed postictal bradycardia. It takes 5 to 10 minutes from the onset of seizure activity until the cardiac situation returns to its pre-existing equilibrium [7].

Neither the cannabinols nor the ephedrine were seen as plausible causes of the terminal asystole in this case. However, the cardiomegaly was.

The routine pre-ECT work-up failed to alert either the psychiatrist or the anesthesiologist to the patient's cardiac condition. Neither a reported history of hypertension nor the obvious obesity were seen as reasons to initiate a more invasive cardiac assessment such as an echocardiogram or an MRI.

The use of an over-the-counter stimulant like ephedrine has been reported, in one case report, to be associated with cardiomyopathy [8], but in the present case, its use followed an extensive history of daily use of methamphetamine and cocaine. Combined, the end result of substantial cardiomegaly appears plausible. This in turn would explain increased vulnerability to the autonomic influence of the extrinsic and intrinsic sympathetic and parasympathetic agents associated with general anesthesia and ECT, and ultimately the refractory, fatally progressive bradycardia. We submit that in patients with a history of substantial and extended abuse of stimulant drugs, the pre-ECT evaluation should include a more thorough cardiac examination than is currently routine. Volumetric measures such as an echocardiogram may well need to be integrated as a standard intervention.

\section{References}

1. McDonald WM, Thompson TR, McCall WV, et al. Electroconvulsive therapy. In Schatzberg AF and Nemeroff CB (eds) Textbook of psychopharmacology. (3rd Edn). Washington DC: American Psychiatric Publishing (2004): 685. 
2. Kramer B. Use of ECT in California 1977-1983. Am J Psychiatry 142 (1985):1190-1192.

3. American Psychiatric Association Task Force on Electroconvulsive Therapy. The practice of electroconvulsive therapy: Recommendations for treatment, training and privileging. Task Force Report on ECT. Washington DC: American Psychiatric Association (2001).

4. Sackeim HA. Memory and ECT: From polarization to reconciliation, JECT 16 (2000): 87-96.

5. Welch CA and Drop LJ. Cardiovascular effects of ECT. Convuls Ther 5 (1989): 35-43.

6. Khan A, Nies A, Johnson G, et al. Plasma catecholamines and ECT. Biol Psychiatry 20 (1985): $799-804$.

7. Welch CA: Electroconvulsive therapy. In Stern TA, Rosenbaum JF, et al. Massachusetts General Hospital comprehensive clinical psychiatry. Philadelphia: Mosby Elsevier (2008): 638.

8. To LB, Sangster JF, Rampling D, et al. Ephedrine-induced cardiomyopathy. Med J Aust 12 (1980): 35-36.

(C) This article is an open access article distributed under the terms and conditions of the
Breative Commons Attribution (CC-BY) license 4.0 